\title{
First report of canine bufavirus in India
}

\author{
Vishweshwar Kumar Ganji ${ }^{1}$ Bhagyalakshmi Buddala ${ }^{1} \cdot$ Narasimha Reddy Yella $^{1} \cdot$ Kalyani Putty $^{1}$ (i)
}

Received: 8 November 2021 / Accepted: 16 January 2022 / Published online: 2 March 2022

(c) The Author(s), under exclusive licence to Springer-Verlag GmbH Austria, part of Springer Nature 2022

\begin{abstract}
Canine bufavirus $(\mathrm{CBuV})$, a novel protoparvovirus of dogs that is associated with enteric and respiratory symptoms, has been reported only in Italy and China. The enteric prevalence of $\mathrm{CBuV}$ in India was investigated, and the nearly complete genome sequence (4292 bp) was amplified and reconstructed for one strain. A nucleotide sequence alignment indicated 93.42-98.81\% identity to the other available $\mathrm{CBuV}$ sequences and $70.88-73.39 \%$ and $54.4-54.8 \%$ identity to human bufavirus and canine parvovirus $2(\mathrm{CPV}-2)$, respectively. The current strain is most closely related to Chinese $\mathrm{CBuV}$ strains, which together form an Asian lineage. This first report of the prevalence of $\mathrm{CBuV}$ in India emphasizes the need for further epidemiological surveillance.
\end{abstract}

Keywords Canine bufavirus · Protoparvovirus $\cdot$ Phylogenetics

Bufaviruses (BuVs) are small, non-enveloped, singlestranded DNA viruses that belong to the family Parvoviridae and genus Protoparvovirus [1,2]. The genome ranges in size from 4.3 to $4.8 \mathrm{~kb}$, with complex hairpin structures at the $5^{\prime}$ and $3^{\prime}$ ends. It has two open reading frames (ORFs): ORF1 and ORF2. ORF1 encodes a non-structural protein, and ORF2 encodes a capsid protein [3]. BuVs were first reported in 2012 in faecal samples from a child with acute gastroenteritis [4]. Since then, BuVs have been reported in several other humans, as well as in wild and domestic animals [1, 5, 6-15]. Canine bufavirus $(\mathrm{CBuV})$ was first reported in Italy in 2016 in a litter of three 5-month-old mixed-breed puppies with canine infectious respiratory disease (CIRD) [11] and, more recently, in China [16, 17]. Although the mechanism is still unknown, $\mathrm{CBuVs}$ are known to be associated with canine enteritis [11, 16, 17]. Information on the epidemiology and genetic features of

Handling Editor: Ana Cristina Bratanich

Kalyani Putty

kalyaniputty@gmail.com

1 Department of Veterinary Biotechnology, College of Veterinary Science, PVNRTVU, Hyderabad-30, 500030 Rajendranagar, Hyderabad, India
$\mathrm{CBuVs}$ is rather limited; the only reports of its existence have been from Italy and China [11, 16-18]. To this end, in the current study, we investigated the prevalence of $\mathrm{CBuV}$ in India. For this, fecal samples from dogs presenting with enteritis at veterinary hospitals in Hyderabad, Telangana State, India, were collected. Also, the nearly full-length genome sequence of one strain was determined and characterized, providing a significant reference for further studies on the evolution and epidemiology of $\mathrm{CBuV}$.

A total of 186 fecal samples were collected from dogs (1 month to 10 years old) presenting with clinical gastroenteritis at veterinary hospitals in Hyderabad, Telangana State, India, during 2019-2020. Of these, 138 were from puppies $(<1$ year old), and 48 were from adult dogs $(>1$ year old) (Supplementary Table S1). The fecal samples were collected in sterile centrifuge tubes using rectal swabs and stored at $-20^{\circ} \mathrm{C}$ until used. The rectal swabs were homogenized in $3 \mathrm{ml}$ of $0.1 \mathrm{M}$ PBS ( $\mathrm{pH}$ 7.4) containing antibiotics (100 IU of benzyl penicillin and $100 \mu \mathrm{g}$ of streptomycin sulphate per ml) and centrifuged at $6000 \mathrm{rpm}$ for $10 \mathrm{~min}$ at $4^{\circ} \mathrm{C}$, and the supernatant was filtered through a $0.22-\mu \mathrm{m}$ syringe filter. Viral DNA was extracted using phenol chloroform and isoamyl alcohol as described by Sambrook and Russel [19]. The extracted DNA was stored at $-20^{\circ} \mathrm{C}$ until use. For detection of $\mathrm{CBuV}$, real-time PCR was performed as 
described previously [11]. The CBuV-positive samples were screened for coinfections with other canine enteric viruses, namely, CPV-2 [20], canine adenovirus 2 (CAdV-2) [21], canine distemper virus (CDV) [22], canine coronavirus $(\mathrm{CCoV})$ [23], canine astrovirus $(\mathrm{CAstV})$ [24], and canine rotavirus (CRV) [25]. Of the 186 samples screened by realtime PCR, eight were found to be positive for $\mathrm{CBuV}$, resulting in a prevalence rate of $4.3 \%$. Of these, six samples were from diarrheic puppies ( $<1$ year old), and two samples were from diarrheic adult dogs ( $>1$ year old). Seven of the eight $\mathrm{CBuV}$-positive samples had coinfections with other enteric viruses, including canine parvovirus CPV-2, CAdV-2, and $\mathrm{CAdV}$, and they were negative for $\mathrm{CCoV}, \mathrm{CRV}$, and CDV (Table 1). One of the PCR-positive samples was selected for amplification of the complete $\mathrm{CBuV}$ genome using five different primer pairs as described previously [26], with slight modifications in the reaction conditions (Supplementary Table S2). The amplified PCR products (Supplementary Fig. S1) were purified using a DNA purification kit (QIAGEN, USA), following the manufacturer's instructions, and were sequenced at the sequencing facility of Bioserve, Pvt. Ltd, Hyderabad, India. The PCR products were sequenced twice. The nearly complete genome sequence of $\mathrm{CBuV}$ strain 407/PVNRTVU/2020 was assembled using SeqMan software (DNASTAR). ORFs were identified using the NCBI ORF Finder (https://www.ncbi.nlm.nih.gov/orffinder/), and the sequences were uploaded to the GenBank database (accession number: MZ574435). The sequenced region of the genome was $4292 \mathrm{nt}$ in length, and ORF-1 and ORF-2 were found to be $1917 \mathrm{nt}$ and $2178 \mathrm{nt}$ in length, respectively (Fig. 1). The length of NS1, VP1, and VP2 was $1917 \mathrm{nt}$ (638 amino acids), $2133 \mathrm{nt}$ (710 amino acids), and $1707 \mathrm{nt}$ (568 amino acids), respectively (Fig. 1).

Nucleotide and amino acid sequences were compared by multiple sequence BLAST analysis. A multiple sequence

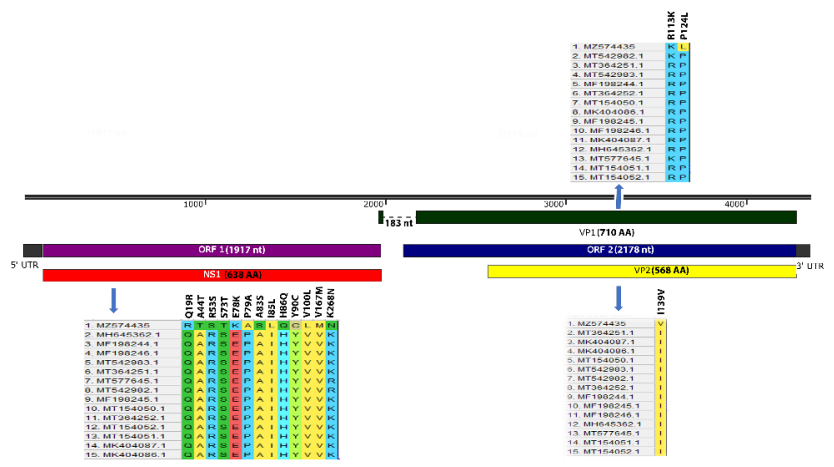

MZ574435 Canine Bufavirus CBuV/2020/PVNRTVU (4292 bp)

Figure 1 Graphical representation of the whole genome sequence of CBuV (MZ574435). Non-synonymous substitutions in NS1, VP1, and VP2 genes are indicated in a format of the actual amino acid followed by the substitution position and the substituted amino acid. alignment of the whole genome was performed using the CLUSTALW algorithm in MEGA X software, and a phylogenetic tree was generated by the maximum-likelihood method with the Tamura 3-parameter model [27]. The reliability of the phylogenetic tree was tested by applying a bootstrap test with 1000 replicates. When compared with the other $\mathrm{CBuV}$ reference strains, the nucleotide sequence identity of the complete genome was $93.42-98.81 \%$ (Supplementary Table S3a). The nucleotide sequence identity in the NS1, VP1, and VP2 coding regions was 96.48-98.13\%, 89.91-99.48\%, and 88.19-99.47\%, respectively (Supplementary Table S3b), and the amino acid sequence identity in the NS1, VP1, and VP2 proteins was $94.98-97.81 \%$, 94.79-99.72\%, and 94.19-99.82\%, respectively (Supplementary Table S3b). Various novel mutations were observed in the NS1, VP1, and VP2 coding regions, with 23, eight, and seven synonymous mutations and 13 , two, and one nonsynonymous substitutions, respectively (Fig. 1). Compared to the other members of the family Parvoviridae including other $\mathrm{CBuVs}$, the nucleotide sequence identity of the complete genome sequence obtained in the current study was $39.5-98.81 \%$ (Supplementary Table S3a). Phylogenetic analysis performed with the nearly complete genome sequence from this study, other publicly available $\mathrm{CBuV}$ sequences, and other protoparvovirus sequences available in the GenBank database showed that the $\mathrm{CBuV}$ isolate from India was most closely related to Chinese strains, and together they formed an Asian lineage (Fig. 2a). Furthermore, the $\mathrm{CBuVs}$ showed the closest relationship to bat and sea otter parvoviruses and all other bufaviruses (human, porcine, rat, and megabat), whereas the canine and avian parvoviruses together formed a separate branch (Fig. 2b).

The recombination analysis tool (RAT) and recombination detection program (RDP) package Beta 4.101 were used for identification of recombinant sequences in default mode. A recombination event identified with a significance of $p<0.01$ by at least four of the nine selected algorithms: RDP, GENECONV, Boot-Scan, Maxchi, Chimaera, SiScan, 3Seq, LARD, and PhylPro, was considered reliable [28]. RAT is a distance-based method of recombination detection [29]. To investigate whether recombination has played a role in $\mathrm{CBuV}$ evolution, $\mathrm{CPVs}, \mathrm{CBuVs}$, HuBuVs, PoBuVs, megabat $\mathrm{BuV}$, rat $\mathrm{BuV}$, bat $\mathrm{BuV}$, avian $\mathrm{BuV}$, human cutavirus, and sea otter parvovirus were analyzed using multiple algorithms in RAT and RDP software. Although no recombination events could be found in the CBuVs, it should be noted that major recombination events possibly drove evolution of other BuVs in the genus Protoparvovirus (Supplementary Fig. S2).

$\mathrm{CBuV}$ is a novel protoparvovirus that was first identified during an outbreak of CIRD [11]. Since then, only a handful of studies from a few countries (Italy, Hungary, and China) 

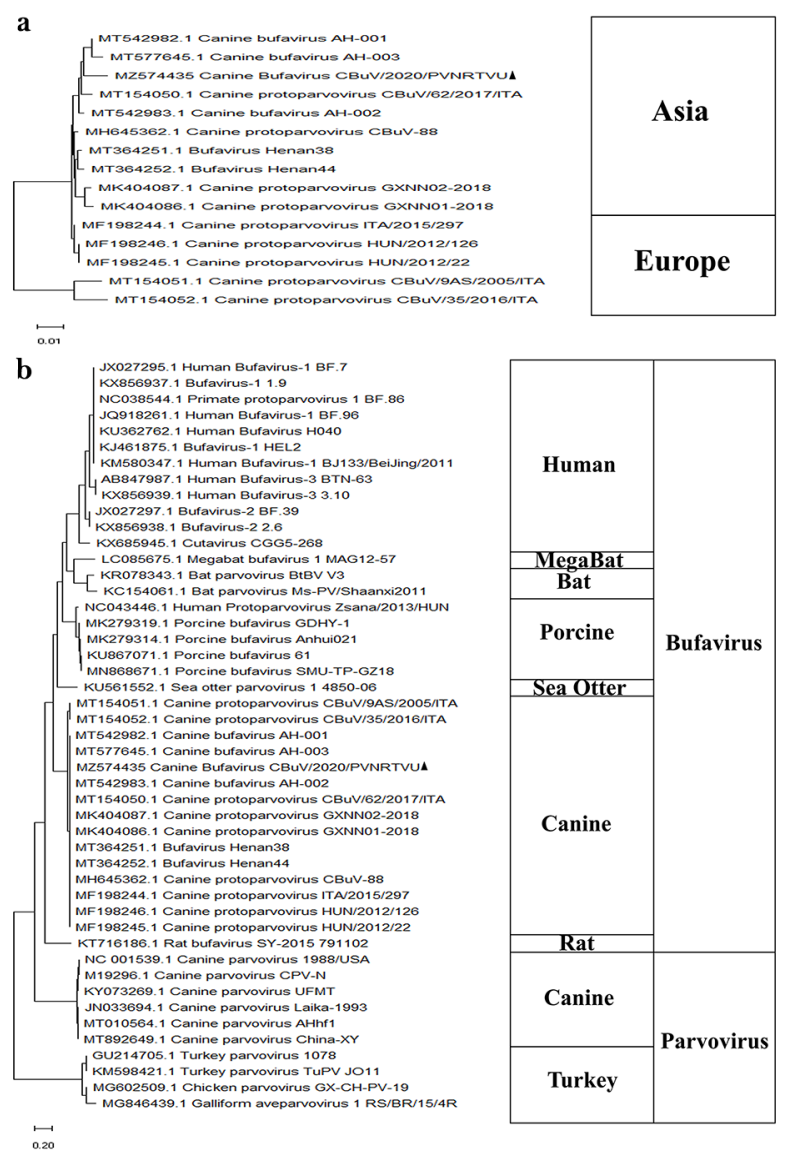

Figure 2 Evolutionary analysis of CBuV (MZ574435). (a) Phylogenetic tree based on whole genome sequences of CBuVs. A total of 15 whole-genome sequences of $\mathrm{CBuV}$ strains published earlier in GenBank were used as references to reconstruct the phylogenetic tree by the maximum-likelihood method with 1000 bootstrap replicates and the Tamura 3-parameter model. The strain from this study is indicated by a filled triangle. (b) Phylogenetic tree of members of the family Parvoviridae based on whole genome sequence. A total of 46 wholegenome sequences of strains infecting different species obtained from the GenBank database were used as references to reconstruct the phylogenetic tree by the maximum-likelihood method with 1000 bootstrap replicates and the Tamura 3-parameter model. The strain from this study is indicated by a filled triangle.

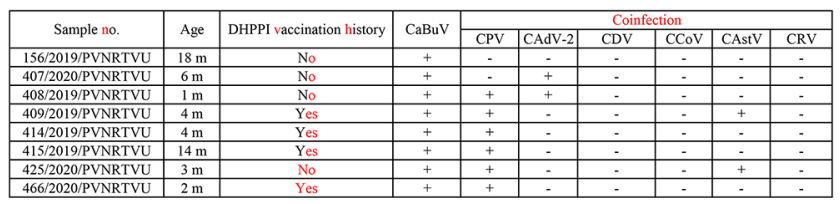

Table 1 Detection of $\mathrm{CBuV}$ in fecal samples and coinfection with $\mathrm{CPV}, \mathrm{CAdV}-2, \mathrm{CDV}, \mathrm{CCoV}$, and CAstV

have reported its prevalence $[11,16-18]$. Here, we report the prevalence of $\mathrm{CBuV}$ in India for the first time. Varying prevalence rates have been reported for this virus so far, ranging from $1.74-42.15 \%[11,16-18,30]$. In our study, we found the prevalence rate to be $4.3 \%$. It has been suggested that geographical and temporal factors as well as the target canine population included in the studies can affect the prevalence rates [18]. A statistically non-significant correlation of $\mathrm{CBuV}$ with enteritis has been reported before; although non-significant, the authors observed higher loads of $\mathrm{CBuV}$ in diarrheic samples than in controls [11, 18]. Moreover, our study, along with another study in which the prevalence of $\mathrm{CBuV}$ was studied extensively in diarrheic dogs, stresses the role of $\mathrm{CBuV}$ in causing enteritis [16]. We found that seven out of eight $\mathrm{CBuV}$-positive samples had coinfections with other enteric viruses, with CPV-2 being the predominant coinfecting virus (6/8), followed by CAstV (2/8) and CAdV-2 (2/8). Similar to our findings, a few other studies also showed CPV-2 to be the major coinfecting agent with $\mathrm{CBuV}$, with the prevalence of $\mathrm{CPV}-2$ ranging from $87.5-100 \%[17,18]$. CPV-2 is a well-known viral agent that is associated with severe gastroenteritis in dogs, especially pups [31]. Further pathogenicity studies will help to clarify the role of coinfections on the severity of gastroenteritis in dogs. We have determined the nearly complete genome sequence of one strain of $\mathrm{CBuV}$ detected in an Indian dog population. Comparison with other publicly available $\mathrm{CBuV}$ sequences revealed NS1 to be the most conserved gene and VP2 to be the most variable, both at the level of nucleotides (NS1: 96.48\%-98.13\%; VP2: 88.19\%99.47\%) and amino acids (NS1: 94.98\%-97.81\%; VP2: 94.19\%-99.82\%). Phylogenetic analysis based on whole genome sequences showed that the current $\mathrm{CBuV}$ strain clustered with $\mathrm{CBuV}$ strains identified in China. Our analysis differentiates all of the available $\mathrm{CBuV}$ sequences into two groups: Asian and European. Like other DNA viruses, parvoviruses have a low mutation rate [17]. The genetic grouping observed in the current study indicates unique evolutionary forces driving evolution of $\mathrm{CBuVs}$. Furthermore, CBuV 407/2020/PVNRTVU showed the highest nucleotide sequence identity $(77.89 \%)$ to a megabat bufavirus strain, followed by bat parvovirus (70.34-79.5\%), porcine bufavirus (73.16-73.4\%), human bufavirus (70.92-73.39\%), sea otter parvovirus $(72.7 \%)$, rat bufavirus $(63 \%)$, canine parvovirus (54.4-54.8\%), and avian parvovirus (39.5-40\%). Individual pairwise comparisons of the NS1, VP1, and VP2 coding regions of 407/2020/PVNRTVU revealed the presence of 13, two, and one unique non-synonymous substitutions when compared to other available $\mathrm{CBuV}$ sequences. Future experimental studies investigating the biological significance of these mutations can elucidate their role (if any) in driving the evolution of this virus and adaptation to local host populations.

In summary, this is the first report of the prevalence and complete genome sequence of a $\mathrm{CBuV}$ strain from India. The Indian strain was found to be related to $\mathrm{CBuVs}$ strains 
from China. The current study forms a foundation for further studies on the epidemiology and genetic diversity of $\mathrm{CBuV}$ in India.

Supplementary Information The online version contains supplementary material available at https://doi.org/10.1007/s00705022-05398-7.

Acknowledgements We wish to express our gratitude to the animal owners who participated in the collection of rectal swab specimens.

Authors' contributions KP conceived and designed the study. VG and BBL performed the experiments. KP, VG, and YNR analyzed the data and wrote the manuscript. The authors read and approved the final manuscript.

Funding The authors acknowledge the Department of Biotechnology (No. BT/ADV/Canine Health/TANUVAS), India, for the financial support.

\section{Declaration}

Ethical approval This research did not involve any human participants or experiments on live animals. All of the work was performed in accordance with standard operating procedures adopted by the Department of Veterinary Biotechnology, PVNRTVU, Hyderabad, India. Before sample collection, the dogs' owners were informed of the purpose of this study, and consent was obtained.

Conflict of interest The authors declare no competing interests with respect to the research, authorship, and/or publication of this article.

\section{References}

1. Hargitai R, Pankovics P, Kertész AM et al (2016) Detection and genetic characterization of a novel parvovirus distantly related to human bufavirus in domestic pigs. Arch Virol 161:1033-1037. https://doi.org/10.1007/s00705-015-2732-4

2. Huang H, Li Y, Wang W et al (2020) Detection and molecular characterization of novel porcine bufaviruses in Guangxi province. Infect Genet Evol 82:104286. https://doi.org/10.1016/j. meegid.2020.104286

3. Vaïsänen E, Paloniemi M, Kuisma I et al (2016) Epidemiology of two human protoparvoviruses, bufavirus and tusavirus. Sci Rep 6:1-8. https://doi.org/10.1038/srep39267

4. Phan TG, Vo NP, Bonkoungou IJO et al (2012) Acute Diarrhea in West African Children: Diverse Enteric Viruses and a Novel Parvovirus Genus. J Virol 86:11024-11030. https://doi.org/10.1128/ jvi.01427-12

5. Altay A, Yahiro T, Bozdayi G et al (2015) Bufavirus genotype 3 in Turkish children with severe diarrhoea. Clin Microbiol Infect 21:965.e1-e4. https://doi.org/10.1016/j.cmi.2015.06.006

6. Huang DD, Wang W, Lu Q, Bin et al (2015) Identification of Bufavirus-1 and Bufavirus-3 in Feces of Patients with Acute Diarrhea, China. Sci Rep 5:13272. https://doi.org/10.1038/srep13272

7. Smits SL, Schapendonk CME, van Beek J et al (2014) New viruses in idiopathic human diarrhea cases, the Netherlands. Emerg Infect Dis 20:1218-1222. https://doi.org/10.3201/eid2007.140190

8. Yahiro T, Wangchuk S, Tshering K et al (2014) Novel human bufavirus genotype 3 in children with severe diarrhea,
Bhutan. Emerg Infect Dis 20:1037-1039. https://doi.org/10.3201/ eid2006.131430

9. Diakoudi G, Lanave G, Capozza P et al (2019) Identification of a novel parvovirus in domestic cats. Vet Microbiol 228:246-251. https://doi.org/10.1016/j.vetmic.2018.12.006

10. Handley SA, Thackray LB, Zhao G et al (2012) Pathogenic simian immunodeficiency virus infection is associated with expansion of the enteric virome. Cell 151:253-266. https://doi.org/10.1016/j. cell.2012.09.024

11. Martella V, Lanave G, Mihalov-Kovács E et al (2018) Novel Parvovirus Related to Primate Bufaviruses in Dogs. Emerg Infect Dis 24:1061. https://doi.org/10.3201/EID2406.171965

12. Melegari I, Di Profio F, Palombieri A et al (2019) Molecular detection of canine bufaviruses in wild canids. Arch Virol 164:2315-2320. https://doi.org/10.1007/s00705-019-04304-y

13. Sasaki M, Orba Y, Anindita PD et al (2015) Distinct lineages of bufavirus in wild shrews and nonhuman primates. Emerg Infect Dis 21:1230-1233. https://doi.org/10.3201/eid2107.141969

14. Sasaki M, Gonzalez G, Wada Y et al (2016) Divergent bufavirus harboured in megabats represents a new lineage of parvoviruses. Sci Rep 201661 6:1-8. https://doi.org/10.1038/srep24257

15. Yang S, Liu D, Wang Y et al (2016) Bufavirus Protoparvovirus in feces of wild rats in China. Virus Genes 52:130-133. https://doi. org/10.1007/s11262-015-1262-1

16. Li J, Cui L, Deng X et al (2019) Canine bufavirus in faeces and plasma of dogs with diarrhoea, China. Emerg Microbes Infect $8: 245-247$

17. Sun W, Zhang S, Huang $H$ et al (2019) First identification of a novel parvovirus distantly related to human bufavirus from diarrheal dogs in China. Virus Res 265:127-131. https://doi. org/10.1016/j.virusres.2019.03.020

18. Di Martino B, Sarchese V, Di Profio F et al (2021) Genetic heterogeneity of canine bufaviruses. Transbound Emerg Dis 68:802812. https://doi.org/10.1111/tbed.13746

19. Sambrook J, Russell DW (2001) Molecular cloning: a laboratory manual. Cold Spring Harbor Laboratory Press

20. Decaro N, Elia G, Martella V et al (2005) A real-time PCR assay for rapid detection and quantitation of canine parvovirus type 2 in the feces of dogs. Vet Microbiol 105:19-28. https://doi. org/10.1016/j.vetmic.2004.09.018

21. Balboni A, Dondi F, Prosperi S, Battilani M (2015) Development of a SYBR Green real-time PCR assay with melting curve analysis for simultaneous detection and differentiation of canine adenovirus type 1 and type 2. J Virol Methods 222:34-40. https:// doi.org/10.1016/j.jviromet.2015.05.009

22. Elia G, Decaro N, Martella V et al (2006) Detection of canine distemper virus in dogs by real-time RT-PCR. J Virol Methods 136:171-176

23. Decaro N, Pratelli A, Campolo M et al (2004) Quantitation of canine coronavirus RNA in the faeces of dogs by TaqMan RTPCR. J Virol Methods 119:145-150. https://doi.org/10.1016/j. jviromet.2004.03.012

24. Martella V, Moschidou P, Lorusso E et al (2011) Detection and characterization of canine astroviruses. J Gen Virol 92:1880 1887. https://doi.org/10.1099/vir.0.029025-0

25. Logan C, O'Leary JJ, O'Sullivan N (2006) Real-time reverse transcription-PCR for detection of rotavirus and adenovirus as causative agents of acute viral gastroenteritis in children. J Clin Microbiol 44:3189-3195. https://doi.org/10.1128/JCM.00915-06

26. Wang Y, Guo X, Zhang D et al (2020) Genetic and phylogenetic analysis of canine bufavirus from Anhui Province, Eastern China. Infect Genet Evol 86:104600. https://doi.org/10.1016/J. MEEGID.2020.104600

27. Kumar S, Stecher G, Li M et al (2018) MEGA X: Molecular evolutionary genetics analysis across computing platforms. Mol Biol Evol 35:1547-1549. https://doi.org/10.1093/molbev/msy096 
28. Martin DP, Murrell B, Golden M et al (2015) RDP4: Detection and analysis of recombination patterns in virus genomes. Virus Evol 1(1):vev003.https://doi.org/10.1093/VE/VEV003

29. Etherington GJ, Dicks J, Roberts IN (2005) Recombination Analysis Tool (RAT): a program for the high-throughput detection of recombination. Bioinformatics 21:278-281. https://doi. org/10.1093/BIOINFORMATICS/BTH500
30. Shao R, Zheng F, Cai S et al (2020) Genomic sequencing and characterization of a novel group of canine bufaviruses from Henan province, China. Arch Virol 165:2699-2702. https://doi. org/10.1007/s00705-020-04785-2

31. Nandi S, Kumar M (2010) Canine parvovirus: Current perspective. Indian J Virol 21:31-44

Publisher's Note Springer Nature remains neutral with regard to jurisdictional claims in published maps and institutional affiliations. 